\title{
C) ACTIVIDADES DEL INSTITUTO
}

\section{SEGUNDO CURSO DE HABILITACION DE INTER- VENTORES DE FONDOS DE 5.* CATEGORIA DE ADMINISTRACION LOCAL}

Después de realizadas las pruebas finales, el día 16 de junio terminó el segundo y último curso de habilitación de Interventores de Fondos de 5. ${ }^{\mathrm{a}}$ categoria de Administración local.

En la mañana de dicho día tuvo lugar, en el Aula Magna del Instituto, la entrega de Títulos a los 45 alumnos que constituyen la Promoción, y a uno que, procedente de la 5.a, y después de seguir un curso de habilitación, pasa a la 3." categoría. El acto fué presidido por el Director general de Administración Local, D. José Luis Moris Marrodán; el Director del Instituto, D. Carlos Ruiz del Castillo y Catalán de Ocón, y los Profesores del curso, señores Saura Pacheco, Pérez Botija, Palop Ruiz, De Lamo Nieto y Ballester Ros.

Habló en nombre de la nueva Promoción el número 1 de la misma, D. José Luis Miño Fuǵarolas, quien agradeció, en nombre de todos los alumnos, las enseñanzas recibidas del Profesorado y las atenciones de la Dirección del Instituto en todo momento.

El señor Ruiz del Castillo dirigió la palabra a los nuevos Interventores de Fondos, diciéndoles que, formados con todo amor en las Aulas del Instituto, deberán llevar a los pueblos, con el ejercicio de su función, el sentido constructivo que tienen estas Promociones de funcionarios, que alguna vez podrán volver a la Escuela Nacional de Administración y Estudios Urbanos, en el decurso de los años, a actualizar conocimientos, cuando la función o los cambios legislativos lo aconsejen.

Cerró el acto el señor Director general de Administración Local, que en elocuentes palabras glosó, entre otros puntos, la doble función, contable y asesora, del Interventor de Fondos, matizada y humanizada la rigidez y la frialdad del número por la adecuación del Derecho y, sobre todo, por el criterio acertado del funcionario. 
Exhortó a todos a laborar por el engrandecimiento de España, pues la Administración pública está integrada por todas y cada una de las Administraciones locales de los pueblos, y de sus felices gestiones se obtiene la total eficacia de aquélla.

Los oradores fueron muy aplaudidos.

La relación nominal de alumnos aprobados, según las puntuaciones obtenidas en la oposición y los Cursos, es la siguiente:

1, D. José Luis Miño Fugarolas ; 2, D. Juan Bautista Fabregó Canal; 3, D. Agustín Rivero Ondovilla; 4, D. Vicente José Arnau Bernia; 5, D. Enrique Arderiu Gras; 6, D. José María Coronas Alonso; 7, D. Juan Antonio Díez Zabaleta ; 8, D. Antonio ReyStolle de la Peña; 9, D. Emilio Sanz Supervía; 10, D. Federico Leach Albert; 11, D. Rafael Talavera Quirós; 12, D. Leopoldo Conde Vaciero; 13, D. Jaime Bigas Turú; 14, D. Alberto Martín Gómez ; 15, D. Mariano Marco Tabar ; 16, D: Mario Pifarré Riera; 17, D. José Luis Pérez Azurmendi ; 18, D. Vicente Pueyo García ; 19, D. Miguel Filgueira Louro; 20, D. Francisco Pimentel Rosado ; 21, D. Enrique Bueso Martín ; 22, D. Jovino Antonio Fernández Cid ; 23, D. Salvador Gómez de Arteche Catalina ; 24, D. Felipe Núñez de Frutos; 25, D. José Arroyo Valderrama ; 26, D. Francisco José Blasco Castany; 27, D. José Luis Hernández Blay; 28, don Manuel Pérez Alfonso; 29, D. Santiago Apilluelo Latorre; 30, don Jesús Ferrero Carbajo; 31, D. Juan Borotau Quinquilla ; 32, D. Antonio de Izaguirre y del Pozo; 33, D. Manuel Díaz López; 34, don Manuel Fernández Sousa; 35, D. Pedro Caballero Pastor ; 36, don Evaristo Vallejo Bonet; 37, D. Juan María Moreno Sánchez; 38, don Laureano Corona de la Torre; 39, D. Fernando Durán Gutiérrez; 40, D. Ramón Roca García ; 41, D. Antonio López Raluy; 42, D. Pedro del Nero Peña ; 43, D. Isaac Heras Romanos ; 44, don Angel Reinaldo Muñoz de Dios, y 45, D. Fernando Marco Hualde.

Interventor de Fondos de 3." categoría de Administración local, don Vicente Yáñez Villarnovo, apto. 


\section{CURSO DE PERFECCION.AMIENTO PARA SECRETARIOS DE 2." CATEGORIA DE ADMINISTRACION LOCAL}

Como final del curso de perfeccionamiento del segundo grupo de Secretarios de 2: categoría de Administración local, tuvo lugar, en el Aula Magna del Instituto, la entrega, a los alumnos que lo han seguido, de los Certificados de aprovechamiento, acto que fué presidido por el Director general de Administración Local, D. José Luis Moris Marrodán; el Director del Instituto, D. Carlos Ruiz del Castillo y Catalán de Ocón, y los Profesores del curso.

Hizo uso de la palabra el Director del Centro, expresando su satisfacción por la asiduidad, interés y aprovechamiento con que se han seguido las enseñanzas profesadas, las que sirven para elevar la competencia profesional y el prestigio del Cuerpo, cerrando el acto el señor Moris Marrodän, con un discurso de congratulación y ofrecimiento personal a los cursillistas, para todo cuanto pueda redundar en servicio y beneficio de la Adminịstración local. Hizo notar cómo la siembra de las enseñanzas del Instituto se va extendiendo por todos los pueblos gracias a estos Cursos de perfeccionamiento, aparte de los de habilitación, alcanzándose un creciente prestigio de los Cuerpos de Administración local que, por constituir el elemento permanente de la Administración, son la savia de la continuidad fecunda de la actividad de los Municipios.

Publicamos a continuación la relación de alumnos asistentes, por orden alfabético:

Don Manuel Abella Navarro, D. Luis Aguilar Capapé, D. Marcial Aguirre Martinez, D. Pascual Alfonsa Crespo, D. Juan Aradas Vilar, D. Roque Arriola Aranzamendi, D. Moisés Balabasquer López, don Lorenzo Bigas Martí, D. Victoriano Blanquet Fondevila, D: Alfonso Buendía Montilla, D. Adrián Cantón Payán, D. Jaime Coca Freginals, D. Juan Coll Drudis, D. Luzgérico Corredera Jiménez, don Emilio Cremades Cubel, D. Mariano Chueca Cuartero, D. Esteban Delgado Tessio, D. Maximiano Escribano y Escribano, D. Francisco Escué Mitjáns, D. Benito Estefanía Fernández, D. Eduardo Fernández Eiriz, D. Nicasio Fernández Morcillo,. D. Julio Ferrer Esquerdo, D. Rafael Gardó Vidal, D. José María Garrido Barrera, don Félix Anacleto González, D. Alejandro Gonzalo Rodríguez, 
don Faustino Gutiérrez Díez, D. Francisco Hedo Utrilla. D. Juan José Herrero Iglesias, D. Manuel Limeres Penado, D. Andrés López Collada, D. Alberto Lozano Espert, D. Benigno Martín y Martín, D. Francisco Martínez García, D. Jesualdo Massó Quilez, D. José Mateos de la Fuente, D. José Ramón Mayáns Codina, D.' Antonio Medina Molinero, D. Francisco Mendizábal Ceciaga, D. Pedro Montero Dominguez, D. Bernardo Mostaza Rodríguez, D. Salvio Moya Mena, D. Abdón Navacerrada Heras, D. Luis Negro Láinez, D. Pedro Pons y Tor, D. José Prat Dalmáu, D. Angel Pujol Net, don Germán Pumares Vila, D. José Rodríguez Alonso, D. José Rodríguez Carballo, D. Hipólito Rodríguez González, D. Aquilino Rodriguez de la Iglesia, D. Arístido Rodríguez Jiménez, D. Angel Rubio y Galán, D. Francisco Rueda Alvarez, D. Amador Ruiz Heráiz, D. Pedro Sánchez Moreno, D. Tomás Sánchez Parra, D. Juan Sans Lleixá, D. Efrén de la Serna Guadalupe, D. Ginés Soler y Soler, D. Benito Sopeña Ruiz, D. Perfecto Sulleiro González y don Isaac Villalba Alfaro.

\section{OPOSICION DE ACCESO A LOS CURSOS DE HABILITACION DE SECRETARIOS DE 2.* CATEGORIA DE ADMINISTRACION LOCAL}

Durante los días 3 al 20 de mayo, y por los 81 opositores que aprobaron el segundo, fué practicado el tercer ejercicio de la oposición de acceso a los cursos de habilitación de Secretarios de 2.3 categoría de Administración local, que fué superado por 27, se retiraron 13, y 39 no obtuvieron la puntuación necesaria para alcanzar la aprobación.

La relación nominal de opositores ingresados en la Escuela, por orden de puntuación, es la siguiente:

Don Romualdo Aragón Gaviña, D. Manuel Muñiz Romero, don Miguel Martín Padial, D. Luis Sabando Villanueva, D. Ricardo Echánove Tuero, D. Saturnino Casillas Candeleda, D. José Luis Azcorreta Arrieta, D. Anastasio Vicente Revilla González, D. Salvador Salvago Mora, D. Juan Antonio Noales Riquelme, D. Angel Moreno Roda, D. Gonzalo Arnica Meridiano, D. José Manuel Sánchez Velasco, D. Angel Manuel Galindo López, D. Francisco López Merino, D. Francisco Trull Roset, D. Rafael Gil Burgués, D. Manuel 
Llorente Toro, D. Roberto Suárez Fernández, D. Benito González Pérez, D. Antonio Martín Sánchez, D. Faustino Delgado Sánchez, don Javier Arumi Dou, D. Pelayo Romero Martín, D. Jesús Bernal Alvarez, D. Julio Padilla Benavides y D. Juan María Jusué González.

Se convoca a los señores comprendidos en la anterior relación para el día 7 de octubre próximo venidero, en la Secretaría General del Instituto, fecha en que dará comienzo el primer curso de habilitación de Secretarios de 2." categoría de Administración local.

A las once horas de dicho día serán recibidos los alumnos por el Ilmo. Sr. Director del Instituto.

En la misına mañana podrán abonar la matrícula y justificar la. posesión del Título exigido en la Convocatoria, o el abono de los derechos del mismo aquellos que no lo tengan justificado.

\section{CONVOCATORIA PARA EL PRIMER CURSO \\ DE HABILITACION DE: DEPOSITARIOS \\ DE FONDOS DE 5. ${ }^{\text {C CATEGORIA }}$ \\ DE ADMINISTRACION LOCAL}

Se convoca a los señores que aprobaron la última oposición celebrada, para el día 7 de octubre próximo venidero, en la Secretaría General del Instituto; para dar comienzo el primer curso de habilitación de Depositarios de Fondos de 5." categoría de Administración local.

A lás once horas de dicho día serán recibidos los alumnos por el Ilmo. Sr. Director del Instituto.

En la misma mañana podrán abonar la matrícula y justificar la posesión del Título de Perito Mercantil, o el abono de los derechos del mismo aquellos que no lo tengan justificado.

\section{PRIMER ICURSO DE LA PRIMERA PROMOCION DE ASPIRANTES AL DIPLOMA DE TECNICO AUXILIAR APAREJADOR DE URBANISMO}

Durante los días 3 al 14 de junio han tenido lugar en el Instituto los períodos escolares cuarto y quinto, refundidos, del primer curso 
de la I Promoción de Aspirantes al Diploma de Técnico Auxilias Aparejador de Urbanismo, con arreglo al siguiente cuadro de clases:

\begin{tabular}{|c|c|c|c|}
\hline Días & Horas & MATERIAS & PROFESORES \\
\hline 3 & $\begin{array}{ll}4.00 a & 5.00 \\
5.00 a & 6.00\end{array}$ & $\begin{array}{l}\text { Legislación urbanistica. } \\
\text { Reparcelación. }\end{array}$ & $\begin{array}{l}\text { Don M. Sánchez Conde } \\
\bowtie \text { J. R. Caso Corral. }\end{array}$ \\
\hline 4 & 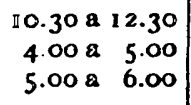 & $\begin{array}{l}\text { Reparcelación. } \\
\text { Información urbanistica. } \\
\text { Información para Expropiaciones. }\end{array}$ & $\begin{array}{l}\text { J. R. Caso Corral. } \\
\text { L. Alemany Soler. } \\
\text { V. Lopez Morales. }\end{array}$ \\
\hline 5 & $\begin{array}{ll}4002 & 5.00 \\
5.002 & 6.00\end{array}$ & $\begin{array}{l}\text { Legislación urbanistica. } \\
\text { Précticas de Valoraciones. }\end{array}$ & $\begin{array}{l}\text { ¿ M. Sánchez Conde. } \\
\text { F. Moreno López. }\end{array}$ \\
\hline 6 & \begin{tabular}{rr|}
$10.30 \mathrm{a}$ & 12.30 \\
$4.00 \mathrm{a}$ & 5.00 \\
$5.00 \mathrm{a}$ & 6.00
\end{tabular} & $\begin{array}{l}\text { Información para Expropiaciones. } \\
\text { Reparceiación. } \\
\text { Información para Expropiaciones. }\end{array}$ & $\begin{array}{l}\text { V. López Morales. } \\
\text { J. R. Caso Corral. } \\
\text { V. López Morales. }\end{array}$ \\
\hline 7 & $\begin{array}{ll}4.00 a & 5.00 \\
5.00 a & 6.00\end{array}$ & $\begin{array}{l}\text { Legislación urbanistica. } \\
\text { Prácticas de Valoraciones. }\end{array}$ & $\begin{array}{l}\text { M. Sánchez Conde. } \\
\text { - F. Moreno López. }\end{array}$ \\
\hline 8 & 10.30212 .30 & Información para Expropiaciones. & »V. López Morales. \\
\hline 30 & \begin{tabular}{rr|}
10.302 & 12.30 \\
$4.00 a$ & 5.00 \\
$5.00 \approx$ & 0.00
\end{tabular} & $\begin{array}{l}\text { Información urbanistica. } \\
\text { Legislación urbanística. } \\
\text { Reparcelación. }\end{array}$ & $\begin{array}{l}\text { L. Alemany Soler. } \\
\text { ¿ M. Sánchez Conde. } \\
\text { J. R. Caso Corral. }\end{array}$ \\
\hline 31 & \begin{tabular}{rr|}
10.302 & 12.30 \\
4.002 & 5.00 \\
5.008 & 6.00
\end{tabular} & $\begin{array}{l}\text { Reparcelación. } \\
\text { Infor:nación urbanistica: } \\
\text { Información para Expropiaciones. }\end{array}$ & $\begin{array}{l}\text { J. R. Caso Corral. } \\
\text { L. Alemany Soler. } \\
\text { V. Lopez Morales. }\end{array}$ \\
\hline 12 & $10.30 \mathrm{a} 12.30$ & Recapitulación. & \\
\hline 33 & 10.30012 .30 & Exámenes. & \\
\hline $\mathbb{1} 4$ & 10.30032 .30 & Exámenes. & \\
\hline
\end{tabular}

Terminados los exámenes, la relación nominal de alumnos declarados aptos para pasar al segundo curso, dividiéndolos, de acuerdo con sus méritos, en tres grupos $y$, dentro de cada grupo, por orden alfabético de apellidos, se formula así:

\section{Grupo primero:}

Herrero Peláez (D. Marcelino), Luque Rojo (D. Manuel), Martinez Pascual (D. Manuel), Mauri Corbella (D. Jaíae), Río Garcia (D. Vicente de?) y Torres Revilla (D. Juan). 


\section{Grupo segundo:}

Anglada y Rosich (D. José), Calvet Serra (D. Ricardo), Cámara Alvarez (D. Enrique), Delgado Serrano (D. José), Gallego Valiente (D. Juan-Angel), Garcia Moreno (D. José-María), Gómez González (D. Juan), Hernández Espinosa (D. Cristóbal), Hernández Sánchez (D. José), Herrero Paredes (D. Juan-Manuel), Jorreto Cifré (don Rafael), Medina Martinez (D. Jesús), Prieto Valtueña (D. Tomás), Ramos Martin (D. Eugenio), Rodriguez del Palacio (L: Tirso) y Villoro Agell (D. Juan).

\section{Grupo tercero:}

Cacheiro Pérez (D. Manuel), Franquesa Millás (D. Melitón), Garrido Callejas (D. Miguel) y Méndez Alonso (D. Gregorio).

Oportunamente les será comunicada la fecha en que se iniciará el primer periodo escolar del segundo curso.

\section{BIBLIOTECARIO DEL INSTITUTO}

Ha sido nombrado Bibliotecário del Instituto D. Vicente Sánchez Muñoz, Licenciado en Filosofía y Letras y del Cuerpo Auxiliar de Archivos, Bibliotecas y Museos.

El señor Sánchez Muñoz ya venía prestando servicios de Colaborador del Instituto desde hace muchos años en funciones análogas a kas que ahora desempeñará.

VISITA AL INSTITUTO DEL DIRECTOR EJECUTIVO DEL INTERNATIONAL CITY MANAGERS ASSOCIATION, MR. NOLTING

El día 10 de mayo visitó el Instituto de Iistudios de Administración Local el Director Ejecutivo del International City Managers Association, Mr. Orin F. Nolting, siendo recibido por el Director, señor Ruiz del Castillo, a quien acompañaba el Secretario generå del mismo, señor Gallego y Burín. 
Míster Nolting recorrió detenidamente las dependencias del Instisuto, informándose del funcionamiento de sus servicios, especialmente de las características de la Escuela Nacional de Administrasion y Estudios Urbanos.

La distinguida personalidad norteamericana se expresó en términos de elogio respecto de la función importantísima que el Instituto de Estudios de Administración Local realiza en el seno de la Administración local española.

La Dirección del Instituto obsequió a Mr. Nolting y a su distinguida esposa, que le acompañaba. en su viaje a Europa, en un típico restaurante madrileño.

\section{OBRA NUEVA}

Estudio de las poblaciones españolas de 20.000 habirans

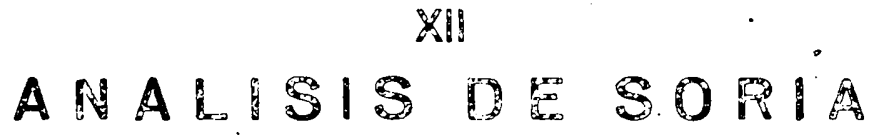

SEMINARIO DE URBANISMO DET. INSTITUTO DF, ESTUDIOS DE ADMINISTRACION LOCAL MADiliD, 1956

Ur volumea con 62 páginas, en folio mayor y al ?áminas en folio mayor y doble folio, con reproducciones fotográficas y plaros, llevardo intercalacios en el texto numerosos gráficos $y$ cuadros, recogen completamente todas las cuestiones urbanas, en un minucioso estudio de la población réferida.

Otros volúrmemes de esta serie:

Precio: 100 pesetas

1. ANAI.ISIS DE AICALA DE HENARES. Precio: 75 pesetas.

1I. ANALISIS DE AVILA. Precio: 25 pesetas.

III y IV. SAGUNTO Y SUECA. Precio: 200 peseras.

V. SEGOVIA. Precio: roo pesetas.

VI. VILLANUEVA Y GELTRU. Precio: 800 pesetas.

VII. SANTA COI.OMA DE GRAMANET. Precio: 70 pesetes.

VIII. CALATAYUD. Precio: 70 pesetas.

IX. ARANJUEZ. Precio: 70 pesetas.

X. AYA.MONIE. Precio: 75 pesetas.

XI. TORRELAVECA. Procio: 100 pesetas.

$P E D I D \cap S A L$

INSTITUTO DE ESTUDIOS DE ADMINISTRACION ZOCAL PUBL: CACIONES Jorquin Garcis Morato, 7.-MADRID 